\title{
Diffeomorphism Invariant Riemannian Framework for Ensemble Average Propagator Computing ${ }^{\star}$
}

\author{
Jian Cheng ${ }^{1,2}$, Aurobrata Ghosh ${ }^{2}$, Tianzi Jiang ${ }^{1}$, and Rachid Deriche ${ }^{2}$ \\ ${ }^{1}$ Center for Computational Medicine, LIAMA, Institute of Automation, \\ Chinese Academy of Sciences, China \\ ${ }^{2}$ Athena Project Team, INRIA Sophia Antipolis - Méditerranée, France \\ jiancheng@nlpr.ia.ac.cn
}

\begin{abstract}
Background: In Diffusion Tensor Imaging (DTI), Riemannian framework based on Information Geometry theory has been proposed for processing tensors on estimation, interpolation, smoothing, regularization, segmentation, statistical test and so on. Recently Riemannian framework has been generalized to Orientation Distribution Function (ODF) and it is applicable to any Probability Density Function (PDF) under orthonormal basis representation. Spherical Polar Fourier Imaging (SPFI) was proposed for ODF and Ensemble Average Propagator (EAP) estimation from arbitrary sampled signals without any assumption.

Purpose: Tensors only can represent Gaussian EAP and ODF is the radial integration of EAP, while EAP has full information for diffusion process. To our knowledge, so far there is no work on how to process EAP data. In this paper, we present a Riemannian framework as a mathematical tool for such task.

Method: We propose a state-of-the-art Riemannian framework for EAPs by representing the square root of EAP, called wavefunction based on quantum mechanics, with the Fourier dual Spherical Polar Fourier (dSPF) basis. In this framework, the exponential map, logarithmic map and geodesic have closed forms, and weighted Riemannian mean and median uniquely exist. We analyze theoretically the similarities and differences between Riemannian frameworks for EAPs and for ODFs and tensors. The Riemannian metric for EAPs is diffeomorphism invariant, which is the natural extension of the affine-invariant metric for tensors. We propose Log-Euclidean framework to fast process EAPs, and Geodesic Anisotropy (GA) to measure the anisotropy of EAPs. With this framework, many important data processing operations, such as interpolation, smoothing, atlas estimation, Principal Geodesic Analysis (PGA), can be performed on EAP data.

Results and Conclusions: The proposed Riemannian framework was validated in synthetic data for interpolation, smoothing, PGA and in real data for GA and atlas estimation. Riemannian median is much robust for atlas estimation.
\end{abstract}

\section{Introduction}

Diffusion MRI (dMRI) is the unique non-invasive technique to study the white matter in vivo by probing the water diffusion. Ensemble Average Propagator (EAP) $P(\mathbf{R})$ is

\footnotetext{
* This work was partially granted by the French Government Award Program, the Natural Science Foundation of China (30730035,81000634), the National Key Basic Research and Development Program of China (2007CB512305), the French ANR "Neurological and Psychiatric diseases" NucleiPark and the France-Parkinson Association.
}

G. Fichtinger, A. Martel, and T. Peters (Eds.): MICCAI 2011, Part II, LNCS 6892, pp. 98-106, 2011.

(C) Springer-Verlag Berlin Heidelberg 2011 
the Probability Density Function (PDF) in $R^{3}$ to describe the diffusion process of water molecules. Under the narrow pulse assumption, $P(\mathbf{R})$ is the Fourier Transform of signal attenuation $E(\mathbf{q})$, i.e. $P(\mathbf{R})=\int E(\mathbf{q}) \exp \left(-2 \pi i \mathbf{q}^{T} \mathbf{R}\right) \mathrm{d} \mathbf{q}, . \mathbf{R}=R \mathbf{r}$ is the displacement vector in $\mathbf{R}$-space, $\mathbf{q}=q \mathbf{u}$ is the reciprocal vector in $\mathbf{q}$-space, $\mathbf{u}$ and $\mathbf{r}$ are unit vectors.

In Diffusion Tensor Imaging (DTI) [4], $P(\mathbf{R})$ is a Gaussian PDF parameterized by its covariance matrix, i.e. tensor. Gaussian distribution is well studied in Information Geometry theory [1]. Riemannian framework [14[13|9] based on Information Geometry was proposed to process the tensor data. Log-Euclidean framework [2] is to approximate Riemannian framework and efficiently process tensors. Riemannian metric for tensors is affine-invariant, while Euclidean metric is not [14]. Riemannian framework and Log-Euclidean framework have proved useful in many works on tensor estimation, interpolation, filtering, segmentation, registration, statistical analysis [14|13|9|2|11].

Since DTI can not deal with complex diffusion process, many methods beyond DTI were proposed to estimate EAP or Orientation Distribution Function (ODF), which is the radial integral of EAP. Recently Spherical Polar Fourier Imaging (SPFI) [3] was proposed to analytically and robustly estimate both ODF and EAP by representing $E(\mathbf{q})$ using Spherical Polar Fourier (SPF) basis. ODF and EAP are both PDFs. Thus it is possible to extend the Riemannian framework from tensors to ODFs and EAPs based on Information Geometry theory. [7]6] and [12] recently developed separately and in parallel a nonparametric Riemannian framework to process ODF data. To our knowledge, recent works for EAPs mainly focus on EAP estimation, and there is no work on how to process EAP data.

In this paper, we first propose the Riemannian framework for EAPs based on the theoretical results in [7[6]. We analyze theoretically the similarities and differences between Riemannian frameworks for EAPs and for ODFs and tensors. For instance the isotropic EAP is not unique, which brings a different definition of Geodesic Anisotropy (GA) as well as the Log-Euclidean framework. Then we implement the framework using the orthonormal basis in SPFI [8]. SPFI provides dSPF basis which analytically obtains GA and Log-Euclidean framework. For the application part, we propose GA for measuring the anisotropy of water diffusion, and some Riemannian operations for EAP computing, such as interpolation, PGA, smoothing, atlas estimation.

\section{Riemannian Framework for EAPs}

In this section we will show Riemannian framework for EAPs and also analyze and compare it with the Riemannian framework for ODFs [8] and highlight the new problems faced and their solutions, since the EAP has different properties from the ODF.

Parametric Family. In quantum mechanics, the square root of the probability of finding the subject at a certain time and position is called as wavefunction. Analogously, the square root of EAP, denoted by $\psi(\mathbf{R})$, is also called as wavefunction. $\psi(\mathbf{R})=$ $\sqrt{P(\mathbf{R})} \geq 0$. Let $\left\{B_{i}(\mathbf{R})\right\}_{i \in N}$ is a given orthonormal basis function set in $R^{3}$ which could sparsely represent $\psi(\mathbf{R})$. then $\psi(\mathbf{R})$ could be represented by finite linear combination of $\left\{B_{i}(\mathbf{R})\right\}$,i.e. $\psi(\mathbf{R} \mid \boldsymbol{c})=\sum_{i=1}^{K} c_{i} B_{i}(\mathbf{R})$, where $\boldsymbol{c}=\left(c_{1}, c_{2}, \ldots, c_{K}\right)^{T}$ is called the Riemannian Coordinate [7]. In practice, we always could choose a large enough $K$ to represent $\psi(\mathbf{R})$, so the assumption here is very weak. Then the Parametric Family or EAP 
space, called $P F_{K}$, could be formulated in (1) [7,6]. Based on $P F_{K}$ in (1), the Parameter Space (PS), denoted as $P S_{k}$, is a subset of sphere $S^{K-1} . P S_{K}=\left\{\boldsymbol{c}\|\boldsymbol{c}\|=\sum_{i=1}^{K} c_{i}^{2}=\right.$ 1, $\left.\sum_{i=1}^{K} c_{i} B_{i}(\mathbf{R}) \geq 0, \forall \mathbf{R} \in R^{3}\right\}$. In Fig. 1(A), we visualize $P S_{3}$ as an example where $\left\{B_{i}\right\}$ were chosen as three piecewise constant orthonormal functions, i.e. three bins for the histogram. Since the formulation in (1) has been well studied in [7]6] and successfully applied for ODFs, in the following we will list the results in [7.6] and modify them if necessary so that they can be applied to EAPs.

$$
P F_{K}=\left\{P(\mathbf{R} \mid \boldsymbol{c})=\psi(\mathbf{R} \mid \boldsymbol{c})^{2}: \psi(\mathbf{R} \mid \boldsymbol{c})=\sum_{i=1}^{K} c_{i} B_{i}(\mathbf{R}) \geq 0, \int P(\mathbf{R} \mid \boldsymbol{c}) \mathrm{d} \mathbf{R}=\|\boldsymbol{c}\|^{2}=1, \forall \mathbf{R} \in R^{3}\right\}
$$

Riemannian Metric. [7] proved that based on formulation in (1), the Riemannian metric [1] is $g_{i j}=\int \frac{\partial \sqrt{P(\mathbf{R} \mid c)}}{\partial c_{i}} \frac{\partial \sqrt{P(\mathbf{R} \mid c)}}{\partial c_{j}} \mathrm{~d} \mathbf{R}=4 \delta_{i j}$. The constant 4 could be ignored, then $P S_{K}$ is a subset of the unit sphere $S^{K-1}$ and the metric is just the Euclidean metric in the sphere. The geodesic distance between two points $P(\mathbf{R} \mid \boldsymbol{c})$ and $P\left(\mathbf{R} \mid \boldsymbol{c}^{\prime}\right)$ will be the angle between them, i.e. $\arccos \left(\boldsymbol{c}^{T} \boldsymbol{c}^{\prime}\right)=\arccos \left(\int_{R^{3}} \psi(\mathbf{R} \mid \boldsymbol{c}) \psi\left(\mathbf{R} \mid \boldsymbol{c}^{\prime}\right) \mathrm{d} \mathbf{R}\right)$. Denote $\boldsymbol{v}_{\boldsymbol{c}}$ is the tangent vector at $\boldsymbol{c}$ towards $\boldsymbol{c}^{\prime}$, then the geodesic, exponential map, logarithmic map all have closed forms. See Fig. 1(A).

$$
\text { Geodesic: } \gamma(t): P(\mathbf{R} \mid \boldsymbol{c}(t)) \text {, where } \boldsymbol{c}(t)=\operatorname{Exp}_{\boldsymbol{c}}\left(t \log _{c}\left(\boldsymbol{c}^{\prime}\right)\right)
$$

$$
\text { Exponential Map: } \operatorname{Exp}_{c}\left(\boldsymbol{v}_{\boldsymbol{c}}\right)=\boldsymbol{c}^{\prime}=\boldsymbol{c} \cos \varphi+\frac{\boldsymbol{v}_{\boldsymbol{c}}}{\left\|\boldsymbol{v}_{\boldsymbol{c}}\right\|} \sin \varphi, \text { where } \varphi=\left\|\boldsymbol{v}_{c}\right\|
$$

$$
\text { Logarithmic Map: } \log _{c}\left(\boldsymbol{c}^{\prime}\right)=\boldsymbol{v}_{\boldsymbol{c}}=\frac{\boldsymbol{c}^{\prime}-\boldsymbol{c} \cos \varphi}{\left\|\boldsymbol{c}^{\prime}-\boldsymbol{c} \cos \varphi\right\|} \varphi, \text { where } \varphi=\arccos \left(\boldsymbol{c}^{T} \boldsymbol{c}^{\prime}\right)
$$

Please note that if we change the orthonormal basis $\left\{B_{i}(\mathbf{R})\right\}$ the exponential map and logarithmic map are invariant under a change of basis matrix, and the geodesic $\gamma(t)$ is also invariant. Different orthonormal basis will obtain equivalent Riemannian framework. The final results of the following Riemannian operations will be the same if the approximation error is negligible. That is why here we consider the formulation using orthonormal basis in [7] instead of the formulation using histogram in [12].

Properties of Parameter Space. [7] showed that $P S_{K}$ for EAPs is a closed convex set of $S^{K-1}$ and it is contained in a convex cone with $90^{\circ}$. See Fig. 11C). [7] also proved for ODFs that "the projection of any $\mathbf{c} \in P S_{K}$ on the Riemannian Coordinate $\mathbf{u}$ of the isotropic ODF should be more than $\frac{1}{\sqrt{4 \pi}}$, if ODFs are less than 1 ", i.e. $\boldsymbol{c}^{T} \boldsymbol{u}=\cos (\boldsymbol{c}, \boldsymbol{u})=$ $\int_{\chi} \sqrt{p}(x \mid c) \frac{1}{\sqrt{|\chi|}} \mathrm{d} x>\frac{1}{\sqrt{|\chi|}} \int_{\chi} p(x \mid \boldsymbol{c}) \mathrm{d} x=\frac{1}{\sqrt{4 \pi}}$. However, It is specific for ODFs. Since the ODF and EAP are both continuous function, it may be more than 1 in some area although the integral in the whole domain is 1 . If $P(\mathbf{R} \mid \boldsymbol{c})>1$, then $\sqrt{P(\mathbf{R})}<P(\mathbf{R})$ and the conclusion will be problematic. Normally for typical ODFs the values are always less than 1 . However, we found that the values of EAPs are normally much larger than 1 because the diffusion time $\tau$ is small. Moreover, the isotropic ODF is unique. While 
the isotropic EAP is not unique, because $P(\mathbf{R})$ is isotropic if $P(R \mathbf{r})=F(R), \forall \mathbf{r} \in S^{2}$ and $F(R)$ could be any positive definite function.

Geodesic Anisotropy (GA), Log-Euclidean Framework. GA for ODFs is defined as the geodesic distance between the ODF and the isotropic one. Log-Euclidean framework for ODFs could be used to approximate Riemannian framework by projecting the ODFs onto the tangent space of the isotropic ODF [7]. However, the isotropic EAP is not unique as discussed above. Please note that the isotropic tensor is not unique in DTI neither. All tensors with three equal eigenvalues are isotropic, i.e. $D=[\lambda, \lambda, \lambda]$. GA for tensors was defined as the geodesic distance from the nearest isotropic tensor [9]. And the identity tensor was chosen and fixed [2] for Log-Euclidean framework. Analogously we define the GA as the geodesic distance between the EAP and the nearest isotropic EAP. It could be proved that for any given EAP with coordinate c, the Riemannian coordinate of the nearest isotropic EAP with the coordinate $\mathbf{u}$, is just the normalized version of the isotropic part of $\mathbf{c}$. We omit the rigorous proof due to page limitations. Furthermore we can also fix a typical isotropic Gaussian distribution for all EAPs. Then Log-Euclidean framework could be obtained by projecting EAPs onto the tangent space of the fixed isotropic EAP. The projection diffeomorphism is defined as $F(\boldsymbol{c})=\log _{\boldsymbol{u}}(\boldsymbol{c})$ where $\boldsymbol{u}$ is the Riemannian coordinate for the fixed isotropic EAP [7]. See Fig. 1]B).

Weighted Mean, Weighted Median and Principal Geodesic Analysis (PGA). Given $N$ EAPs $f_{1}, f_{2}, \ldots, f_{N}$ in $P S_{K}$ and the weight vector $\boldsymbol{w}=\left(w_{1}, w_{2}, \ldots, w_{N}\right)^{T}$ with $\sum_{i=1}^{N} w_{i}=$ $1, w_{i} \in[0,1]$, the weighted Riemannian mean $\mu_{w}$ is defined as the minimizer of the weighted sum of squared distances [14|13|9|7|5]. And the weighted Riemannian median $m_{w}$ is defined as the minimizer of the weighted sum of distances [106].

$$
\mu_{w}=\arg \min _{f \in P S_{K}} \sum_{i=1}^{N} w_{i} d\left(f, f_{i}\right)^{2} \quad m_{w}=\arg \min _{f \in P S_{K}} \sum_{i=1}^{N} w_{i} d\left(f, f_{i}\right)
$$

[76] proved that the weighted Riemannian mean and weighted Riemannian median uniquely exist in the manifold $P S_{K}$ based on the general results in [5[10]. And they can be obtained efficiently from gradient descent method on the manifold. Normalized Euclidean mean is chosen as the initialization, which makes the methods converge fast. See Algorithm 1 and 2. For Log-Euclidean framework, the Riemannian mean has closed form, i.e. $\mu_{w}=F^{-1}\left(\sum_{i=1}^{N} w_{i} F\left(f_{i}\right)\right)$ [7]. When Riemannian mean $\mu$ of $\left\{f_{i}\right\}$ is obtained, we can find some Principal Components (PCs) based on Principal Geodesic Analysis (PGA) [11] by eigen-decomposition of the covariance matrix at $\mu$. If $v$ is one eigenvector with eigenvalue $\lambda_{k}$, then the PC will be $\operatorname{Exp}_{\mu}\left(\alpha_{k} v\right)$, where $\alpha_{k} \in R$ is the mode variation. Normally $\alpha_{k}$ is chosen in $\left[-3 \sqrt{\lambda_{k}}, 3 \sqrt{\lambda_{k}}\right]$ [11]. PGA has been proposed for tensor analysis [11] and for ODF analysis [12]. However PGA in [12] decompose a covariance matrix in a very high dimension, which is much inefficient. Actually we can perform PGA on ODFs and EAPs just in a low dimension via orthonormal basis representation, which will get the same final results as we discussed above.

Diffeomorphism Invariant: A Natural Extension. What is the connection between the proposed metric and the previous metric for tensors [14|13|9]? Actually they are all the Fisher Information metric in Information Geometry. The Fisher information metric is actually diffeomorphism invariant. Denote $P_{X}(x \mid c)$ is a PDF on 

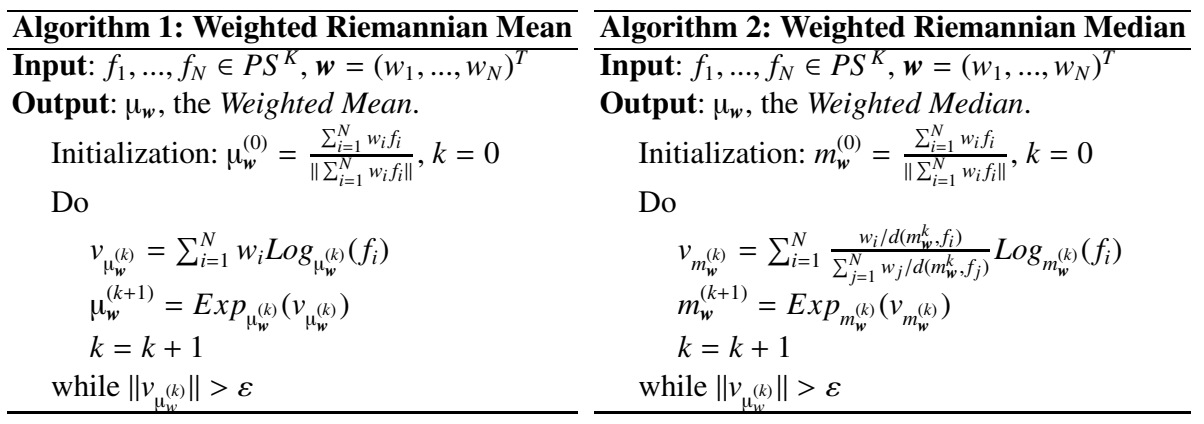

domain $\chi, h: \chi \mapsto \chi$ is a diffeomorphism. $P_{Y}(y \mid c)$ is the PDF under $g$, i.e. $y=$ $h(x)$. Then $P_{Y}(y)=\left|\nabla h^{-1}(y)\right| P_{X}\left(h^{-1}(y)\right)$. By considering $d y=|\nabla h(y)| d x$, we have $g_{i j}=\int_{\chi} \frac{\partial \sqrt{P_{Y}(y \mid c)}}{\partial c_{i}} \frac{\partial \sqrt{P_{Y}(y \mid c)}}{\partial c_{j}} \mathrm{~d} y=\int_{\chi} \frac{\partial \sqrt{P_{X}(x \mid c)}}{\partial c_{i}} \frac{\partial \sqrt{P_{X}(x \mid c)}}{\partial c_{j}} \mathrm{~d} x$, which means the metric $g_{i j}$ is diffeomorphism invariant. So for any two given ODFs or EAPs, the Riemannian distance between them is diffeomorphism invariant. If we constrain the PDF $P_{X}(x \mid \mathbf{D})$ as a Gaussian distribution parameterized by tensor $\mathbf{D}$, then it could be easily proved that $P_{Y}(y \mid \mathbf{D})$ is still Gaussian if and only if $h(x)$ is an affine transform, i.e. $h(x)=A x, A$ is a nonsingular matrix. In this sense the proposed Riemannian metric for EAPs is actually a natural extension of previous affine-invariant metric for tensors [14[139]. The diffeomorphism invariant metric is probably useful in registration.

\section{Implementation via Spherical Polar Fourier Imaging (SPFI)}

In theory, the Riemannian framework for EAPs could be implemented by any orthonormal basis. However, so far there is no direct way to estimate the Riemannian Coordinate $\boldsymbol{c}$ from DWI signals $E(\mathbf{q})$. Existing methods only estimate EAPs. SPFI is a fast, regularized, robust method to estimate EAPs without any assumption [3]8]. In SPFI, $E(\mathbf{q})$ is represented by an orthonormal basis $\left\{B_{n l m}(\mathbf{q})\right\}$ in formula (6), named SPF basis, where $\zeta$ is a fixed scale parameter, $R_{n}(q)$ is the Gaussian-Laguerre function and $Y_{l}^{m}(\mathbf{u})$ is the $l$ order $m$ degree Spherical Harmonic. [8] proved that the EAP $P(\mathbf{R})$ could be analytically obtained in formula (8) from the same coefficients $\left\{a_{n l m}\right\}$, where ${ }_{1} F_{1}$ is the confluent hypergeometric function. $\left\{D_{n l m}(\mathbf{R})\right\}$ is actually an orthonormal basis in $\mathbf{R}$ space, called Fourier dual Spherical Polar Fourier (dSPF) basis, because of Parseval's theorem, i.e. $\delta_{n l m}^{n^{\prime} l^{\prime} m^{\prime}}=\int_{R^{3}} B_{n l m}(\mathbf{q}) B_{n^{\prime} l^{\prime} m^{\prime}}(\mathbf{q}) \mathrm{d} \mathbf{q}=\int_{R^{3}} D_{n l m}(\mathbf{R}) D_{n^{\prime} l^{\prime} m^{\prime}}(\mathbf{R}) \mathrm{d} \mathbf{R}$. So SPFI actually provides two orthonormal basis. One is $\left\{B_{n l m}(\mathbf{q})\right\}$ in $\mathbf{q}$ space for $E(\mathbf{q})$ and the other one is $\left\{D_{n l m}(\mathbf{R})\right\}$ in $\mathbf{R}$ space for $P(\mathbf{R})$.

$$
\begin{gathered}
E(q \mathbf{u})=\sum_{n=0}^{N} \sum_{l=0}^{L} \sum_{m=-l}^{l} a_{n l m} R_{n}(q) Y_{l}^{m}(\mathbf{u}) \quad B_{n l m}(\mathbf{q})=R_{n}(q) Y_{l}^{m}(\mathbf{u}) \\
R_{n}(q)=\kappa_{n}(\zeta) \exp \left(-\frac{q^{2}}{2 \zeta}\right) L_{n}^{1 / 2}\left(\frac{q^{2}}{\zeta}\right) \quad \kappa_{n}(\zeta)=\left[\frac{2}{\zeta^{3 / 2}} \frac{n !}{\Gamma(n+3 / 2)}\right]^{1 / 2} \\
P(R \mathbf{r})=\sum_{n=0}^{N} \sum_{l=0}^{L} \sum_{m=-l}^{l} a_{n, l, m} F_{n l}(R) Y_{l}^{m}(\mathbf{r}) \quad D_{n l m}(\mathbf{R})=F_{n l}(R) Y_{l}^{m}(\mathbf{r})
\end{gathered}
$$




$$
F_{n l}(R)=\frac{\zeta^{0.5 l+1.5} \pi^{l+1.5} R_{0}^{l} \kappa_{n}(\zeta)}{(-1)^{l / 2} \Gamma(l+1.5)} \sum_{i=0}^{n}\left(\begin{array}{c}
n+0.5 \\
n-i
\end{array}\right) \frac{(-1)^{i}}{i !} 2^{0.5 l+i+1.5} \Gamma(0.5 l+i+1.5)_{1} F_{1}\left(\frac{2 i+l+3}{2} ; l+\frac{3}{2} ;-2 \pi^{2} R^{2} \zeta\right)
$$

After obtaining the continuous function $P(\mathbf{R})$ in $(8)$ represented by $\left\{D_{n l m}(\mathbf{R})\right\}$, we can get many discrete samples $\left\{\psi\left(\mathbf{R}_{i}\right)\right\}$ from the wavefunction $\psi(\mathbf{R})=\sqrt{P(\mathbf{R})}$. Then similarly with [7], a least square fitting using the same basis $\left\{D_{n l m}(\mathbf{R})\right\}$ is applied to estimate the Riemannian coordinates $c$ from these samples $\left\{\psi\left(\mathbf{R}_{i}\right)\right\}$. After the Riemannian Coordinate $\boldsymbol{c}=\left\{c_{n l m}\right\}$ is estimated, GA is the distance between $c$ and the nearest isotropic EAP with coordinate $\boldsymbol{u}$, which is the normalized version of isotropic part of $\boldsymbol{c}$. With the basis $\left\{D_{n l m}(\mathbf{R})\right\}$, the isotropic part of $\left\{c_{n l m}\right\}$ is analytically obtained as $\left\{c_{n l m} \delta_{l m}^{00}\right\}$. The isotropic EAP with coordinate $(1,0, \ldots, 0)^{T}$, which is an isotropic Gaussian based on SPF basis in (6), was chosen and fixed for Log-Euclidean framework. Thus LogEuclidean framework can be obtained analytically from the given Riemannian Coordinate thanks to dSPF basis. Moreover we can perform many data processing algorithms, such as interpolation, smoothing etc. [7|12|14].

\section{Applications and Experiments}

Experiments on Diffeomorphism Invariance. Here we give a simple example to demonstrate the diffeomorphism invariance for the Riemannian metric using a affine transform. Mixture of tensors model $P(\mathbf{R})=w_{1} P\left(\mathbf{R} \mid \mathbf{D}_{1}\right)+w_{2} P\left(\mathbf{R} \mid \mathbf{D}_{2}\right)$ was used to generate the synthetic data. $\mathbf{D}_{1}$ and $\mathbf{D}_{2}$ have the same eigenvalues $[1.7,0.3,0.3] \times 10^{-3} \mathrm{~mm}^{2} / \mathrm{s}$ with different directions. We estimated Riemannian Coordinates and computed the Riemannian distance between the EAP in the center and the EAPs in other voxels. Afterwards a given affine transform $A$ was performed on the mixture model, i.e. $(A \circ P)(\mathbf{R})=$ $w_{1} P\left(\mathbf{R} \mid A^{T} \mathbf{D}_{1} A\right)+w_{2} P\left(\mathbf{R} \mid A^{T} \mathbf{D}_{2} A\right)$. With this transformed model, we calculated again the distance maps. In Fig. 1, (C1) shows the original EAPs at $15 \mu \mathrm{m}$ and (C2) shows the transformed EAPs. The distance maps were used to color the glyphs and were set as the background. It is clear that the distance is invariant under the transform. This experiment showed that Riemannian metric for EAPs is diffeomorphism invariant, which is a natural extension of affine-invariant metric for tensors.

Interpolation and PGA. We demonstrate the Lagrange interpolation of EAPs in 1D and 2D in Fig. 1] where the weights are coded by the spatial distance [7]. For 1D case, two EAPs were generated from tensors with eigenvalues $[1.7,0.3,0.3] \times 10^{-3} \mathrm{~mm}^{2} / \mathrm{s}$ and $[0.3,0.3,0.3] \times 10^{-3} \mathrm{~mm}^{2} / \mathrm{s}$. For $2 \mathrm{D}$ case, one EAP was generated from $[0.7,0.7,0.7] \times$ $10^{-3} \mathrm{~mm}^{2} / \mathrm{s}$, the other three from $[1.7,0.3,0.3] \times 10^{-3} \mathrm{~mm}^{2} / \mathrm{s}$ with 3 orthogonal directions. Fig. 1(D1) and (D3) show the results for Riemannian framework and (D2) and (D4) for Log-Euclidean framework. EAPs were visualized at $15 \mu \mathrm{m}$ and the glyphs were colored by GA values. It is clear that the interpolation from Riemannian framework and Log-Euclidean framework have very similar results. The first two principal components of EAP field in Fig. 1(D3) based on PGA are shown in (E1) and (E2). The two components showed clearly the three orthogonal directions as well as the mean EAP. 


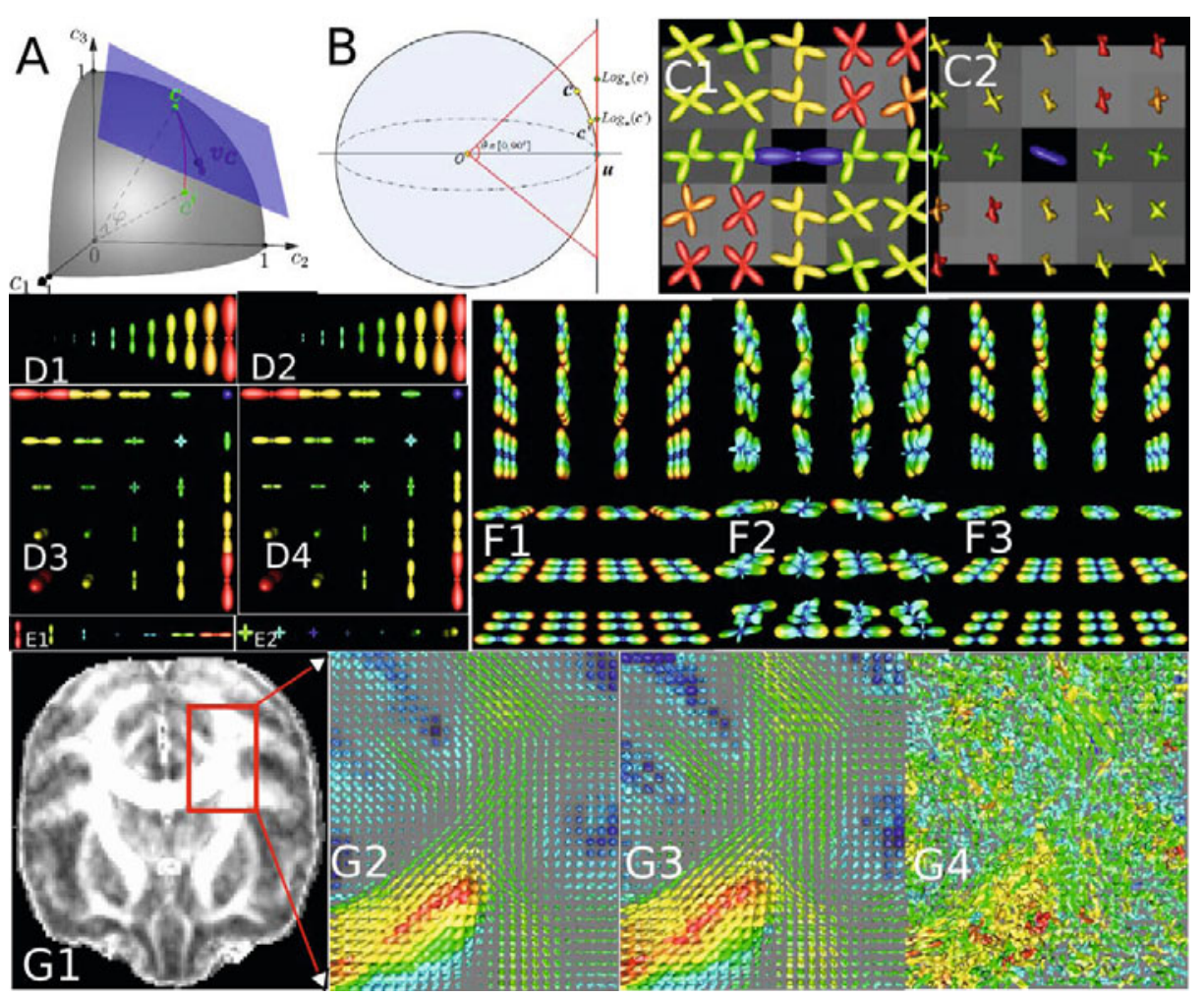

Fig. 1. (A): an example of $P S_{3}$; (B): properties of $P S_{K}$; $(\mathrm{C} 1, \mathrm{C} 2)$ : original EAPs and transformed EAPs; (D1,D2): 1D interpolation via Riemannian framework and Log-Euclidean framework; (D3,D4): 2D interpolation via Riemannian framework and Log-Euclidean framework; (E1,E2): the first two PCs in PGA for EAPs in D3; (F1,F2,F3): original EAPs, noisy EAPs, smoothed EAP; (G1): GA map; $(\mathrm{G} 2, \mathrm{G} 3)$ : original and noisy atlas; $(\mathrm{G} 4)$ : noisy EAPs from one subject;

Smoothing. Actually all filtering algorithms in [14] for tensors and in [6]12] for ODFs could be generalized into EAP field. Here we just demonstrate a simple Gaussian smoothing method. In this method the filtered EAP in every voxel is the weighted mean/median of EAPs in a given neighborhood. The weights were chosen from spatial Gaussian kernel. Fig. 1 $(\mathrm{F} 1)$ shows the ground truth EAP profiles generated from tensor model with eigenvalues $[1.7,0.3,0.3] \times 10^{-3} \mathrm{~mm}^{2} / \mathrm{s}$ EAPs in (F2) were estimated from SPFI from single shell DWI signals with $b=1500 \mathrm{~s} / \mathrm{mm}^{2}$ and $S N R=5$ [8]. The results of Gaussian smooth using Riemannian mean were shown in Fig. 1/F3). The deviation of the Gaussian kernel was set as 1 voxel.

GA and Riemannian Median Atlas Estimation. Riemannian median is more robust than Riemannian mean and Euclidean mean, which makes it more appropriate for atlas estimation [106]. We construct an EAP atlas from five monkey data, with three $b$ values $\left(500 / 1500 / 3000 \mathrm{~s} / \mathrm{mm}^{2}\right), 30$ directions at each shell. Since so far there is no common registration method for EAP data and it is also not our focus here, we just use a naive 
way to align the EAP data. All DWIs from 5 subjects were aligned via affine registration to a template made by non-diffusion weighted images. The affine transformation was used to rotate the gradient directions for each subject through the finite strain method. After that, EAP images were estimated via SPFI from registrated DWIs and reorientated gradient directions. The atlas in every voxel was estimated as the Riemannian median of the five EAP images. And GA map in Fig. 1 (G1) was estimated from the atlas. To test the robustness, we add Rician noise with $S N R=10$ to the DWIs of one subject, then re-estimate the atlas. Fig. 1 showed the noisy EAPs (G4) and the EAPs of the two atlases $(\mathrm{G} 2, \mathrm{G} 3)$ in the red region in $(\mathrm{G} 1)$. The atlas from noisy data is much similar with the one from the real data, which validates the robustness.

\section{Conclusion}

In this paper, we propose a diffeomorphism invariant Riemannian framework for EAP computing, which is a natural extension of previous Riemannian framework for tensors and ODFs. In the proposed framework, exponential map, logarithmic map and geodesic have closed forms, weighted Riemannian mean and median uniquely exist and could be calculated efficiently. We analyze theoretically the similarities and differences between Riemannian frameworks for EAPs and for ODFs and tensors. The isotropic EAP is not unique, which brings a different definition of GA and the Log-Euclidean framework compare to the Riemannian framework for ODFs. The Riemannian framework for EAPs is more like the Riemannian framework for tensors, since we need to define what is the nearest isotropic EAP. And if we constrain the EAP as a Gaussian, the diffeomorphism invariance property will be the affine-invariance for tensors. The proposed Riemannian framework was implemented via SPFI by representing the wavefunction with dSPF basis, which has closed forms for GA and Log-Euclidean framework. For application part, we propose GA to measure anisotropy, Lagrange interpolation, Gaussian smoothing, PGA and median atlas estimation for EAP computing.

\section{References}

1. Amari, S., Nagaoka, H.: Methods of Information Geometry. American Mathematical Society, USA (2000)

2. Arsigny, V., Fillard, P., Pennec, X., Ayache, N.: Log-Euclidean metrics for fast and simple calculus on diffusion tensors. Magnetic Resonance in Medicine 56, 411-421 (2006)

3. Assemlal, H.E., Tschumperlé, D., Brun, L.: Efficient and robust computation of PDF features from diffusion MR signal. Medical Image Analysis 13, 715-729 (2009)

4. Basser, P.J., Mattiello, J., LeBihan, D.: MR diffusion tensor spectroscropy and imaging. Biophysical Journal 66, 259-267 (1994)

5. Buss, S.R., Fillmore, J.P.: Spherical averages and applications to spherical splines and interpolation. ACM Transactions on Graphics 20, 95-126 (2001)

6. Cheng, J., Ghosh, A., Jiang, T., Deriche, R.: Riemannian median and its applications for orientation distribution function computing. In: ISMRM (2010)

7. Cheng, J., Ghosh, A., Jiang, T., Deriche, R.: A riemannian framework for orientation distribution function computing. In: Yang, G.-Z., Hawkes, D., Rueckert, D., Noble, A., Taylor, C. (eds.) MICCAI 2009. LNCS, vol. 5761, pp. 911-918. Springer, Heidelberg (2009) 
8. Cheng, J., Ghosh, A., Jiang, T., Deriche, R.: Model-free and analytical EAP reconstruction via spherical polar fourier diffusion MRI. In: Jiang, T., Navab, N., Pluim, J.P.W., Viergever, M.A. (eds.) MICCAI 2010. LNCS, vol. 6361, pp. 590-597. Springer, Heidelberg (2010)

9. Fletcher, P.T.: Statistical Variability in Nonlinear Spaces Application to Shape Analysis and DT-MRI. Ph.D. thesis, University of North Carolina (2004)

10. Fletcher, P.T., Venkatasubramanian, S., Joshi, S.: The geometric median on riemannian manifolds with application to robust atlas estimation. NeuroImage 45, S143-S152 (2009)

11. Fletcher, P., Joshi, S.: Riemannian geometry for the statistical analysis of diffusion tensor data. Signal Processing 87, 250-262 (2007)

12. Goh, A., Lenglet, C., Thompson, P., Vidal, R.: A nonparametric Riemannian framework for processing high angular resolution diffusion images and its applications to ODF-based morphometry. NeuroImage (2011)

13. Lenglet, C., Rousson, M., Deriche, R.: Statistics on the manifold of multivariate normal distributions theory and application to diffusion tensor MRI processingy. Journal of Mathematical Imaging and Vision 25(3), 423-444 (2006)

14. Pennec, X., Fillard, P., Ayache, N.: A riemannian framework for tensor computing. International Journal of Computer Vision 66, 41-66 (2006) 